\title{
Broadband noncollinear optical parametric amplification without angularly dispersed idler
}

\author{
Shu-Wei Huang, ${ }^{1, *}$ Jeffrey Moses, ${ }^{1}$ and Franz X. Kärtner ${ }^{1,2}$ \\ ${ }^{1}$ Department of Electrical Engineering and Computer Science and Research Laboratory of Electronics, \\ Massachusetts Institute of Technology, Cambridge, Massachusetts 02139, USA \\ ${ }^{2}$ Center for Free-Electron Laser Science, DESY, and Department of Physics, University of Hamburg, \\ Notkestraße 85, D-22607 Hamburg, Germany \\ ${ }^{*}$ Corresponding author: klkla@mit.edu
}

Received March 27, 2012; revised May 20, 2012; accepted May 27, 2012; posted May 30, 2012 (Doc. ID 165534); published July 4, 2012

\begin{abstract}
We report a new scheme for direct generation of broadband angular-dispersion-free mid-IR idler pulses via noncollinear optical parametric amplification when group-velocity matched wavelengths cannot be found and the traditional noncollinear geometry fails to increase the phase-matching bandwidth. The scheme does not require any post-amplification idler angular dispersion compensation. We derive and interpret the condition for broadband amplification and absence of idler angular dispersion. A broadband angular-dispersion-free $2.15 \mu \mathrm{m}$ idler pulse is generated as an experimental demonstration. We identify the potential of the scheme to generate a broadband $3.5 \mu \mathrm{m}$ idler, with a bandwidth supporting a sub-two-cycle pulse. (c) 2012 Optical Society of America
\end{abstract}

OCIS codes: $190.4970,320.7110$.

Broadband mid-IR sources have been an integral part of time-resolved vibrational spectroscopies because this wavelength range is in resonance with vibrational transitions of important bio-molecules such as $\mathrm{O}-\mathrm{H}$ stretching [1] and C-O stretching [2]. Recently, there also has been interest in applying mid-IR sources to strong-field physics research such as the study of photoionization processes [3] and long-wavelength driven high-harmonic generation (HHG) [ㄴ-6].

Due to the lack of mid-IR laser media, differencefrequency generation or optical parametric amplification (OPA) have been largely employed to down-convert visible or near-IR light sources [7-10]. It has been shown that mid-IR crystals such as $\mathrm{LiIO}_{3}, \mathrm{KNbO}_{3}$, and $\mathrm{MgO}: \mathrm{LiNbO}_{3}$ have group-velocity matched wavelengths, when pumped at $800 \mathrm{~nm}$, between $950 \mathrm{~nm}$ and $1050 \mathrm{~nm}$, and thus allow the direct generation of a broadband midIR idler with spectra supporting three-cycle pulses [7]. For wavelengths where signal and idler group velocities $\left(V_{\mathrm{gs}}\right.$ and $\left.V_{\mathrm{gi}}\right)$ are not matched, a noncollinear geometry can be employed so that $V_{\mathrm{gs}}=V_{\mathrm{gi}} \cos \Omega$. This achieves a broad phase-matching bandwidth at the cost of introducing idler angular dispersion [11]. It has been shown that once its angular dispersion is compensated, the idler can also be utilized for experiments [12].

However, there is no guarantee that the condition of the traditional noncollinear OPA, i.e., $V_{\mathrm{gs}}=V_{\mathrm{gi}} \cos \Omega$, can be satisfied and thus broadband amplification is sometimes prohibited. For example, when pumped at $1 \mu \mathrm{m}$, the condition cannot be fulfilled for all signal wavelengths below the degenerate wavelength in mid-IR crystals such as $\mathrm{LiIO}_{3}, \mathrm{KNbO}_{3}$, and $\mathrm{MgO}: \mathrm{LiNbO}_{3}$. The same is true in lithium triborate (LBO) for signal wavelengths shorter than $720 \mathrm{~nm}$, pumping at $523 \mathrm{~nm}$. On the other hand, pumping at $1 \mu \mathrm{m}$ or its second harmonic is advantageous for power and energy scalability because it is compatible with high-power ytterbium $(\mathrm{Yb})$ fiber technology and high-energy Yb:YAG laser technology.

In this Letter, we present a new scheme for direct generation of broadband, angular-dispersion-free mid-IR idler pulses when signal-idler group velocity matching cannot be achieved and the traditional noncollinear geometry fails to increase the phase-matching bandwidth. The conditions for broadband amplification and absence of idler angular dispersion can be fulfilled simultaneously by introducing not only noncollinear geometry but also signal angular dispersion. A broadband (1.8-2.4 $\mu \mathrm{m}$ ), angular-dispersion-free idler pulse, which can be used to seed a $2.15 \mu \mathrm{m}$ optical parametric chirped pulse am-

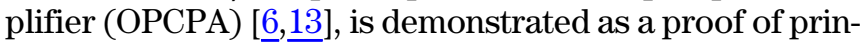
ciple. Furthermore, we identify the potential of the scheme to generate a broadband $3.5 \mu \mathrm{m}$ idler from a $1 \mu \mathrm{m}$ pumped OPCPA, with a bandwidth more than a factor of 2 broader than the current state-of-the-art $[9,10]$.

Figure 1 shows the principle of the scheme and defines the angles $\alpha, \beta$, and $\Omega$ between interacting wavevectors $k_{s}, k_{i}$, and $k_{p}$. To obtain broadband amplification, not only the wavevector mismatch but also its derivative with respective to the signal optical frequency has to be set to zero as shown in Eqs. (1) and (2).

$$
\begin{gathered}
\left\{\begin{array}{l}
\Delta k_{\|}=k_{p} \cos \beta-k_{i}-k_{s} \cos \Omega=0 \\
\Delta k_{\perp}=k_{p} \sin \beta-k_{s} \sin \Omega=0
\end{array}\right. \\
\left\{\begin{array}{l}
k_{p} \sin \beta \frac{d \beta}{d \omega_{s}}+\frac{d k_{i}}{d \omega_{s}}+\frac{d k_{s}}{d \omega_{s}} \cos \Omega-k_{s} \sin \Omega \frac{d \Omega}{d \omega_{s}}=0 \\
k_{p} \cos \beta \frac{d \beta}{d \omega_{s}}-\frac{d k_{s}}{d \omega_{s}} \sin \Omega-k_{s} \cos \Omega \frac{d \Omega}{d \omega_{s}}=0 .
\end{array}\right. \\
\text { (a) }_{\alpha} \text { (b) }
\end{gathered}
$$
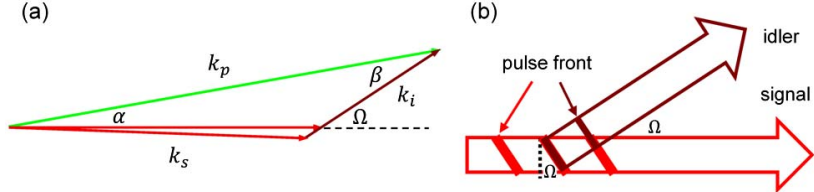

Fig. 1. (Color online) (a) Schematic of the OPA geometry; (b) interpretation of Eq. (3). The signal and the idler will stay effectively overlapped when Eq. (3) is fulfilled. The idler will have no pulse front tilting, and thus no angular dispersion, if Eq. (3) is satisfied. 
With some algebraic manipulations and the constraint that the idler has no angular dispersion, i.e., $d \beta / d \omega=0$, one can reach Eq. (3), which determines the required noncollinear angle and signal angular dispersion $(d \alpha / d \lambda)$ for simultaneous broad phase-matching bandwidth and angular-dispersion-free idler.

$$
\left\{\begin{array}{l}
V_{\mathrm{gi}}=V_{\mathrm{gs}} \cos \Omega, \\
\tan \Omega=\lambda_{s} \frac{V_{\mathrm{gs}}}{V_{\mathrm{ps}}} \frac{d \alpha}{d \lambda},
\end{array}\right.
$$

where $V_{\mathrm{ps}}$ is the signal phase velocity.

The interpretation of Eq. (3) is depicted in Fig. 1(b). Equation (3) means that the required signal-idler angle is the one that makes the idler group velocity equal to the projection of the signal group velocity along the idler propagation direction. Of note, the relation between $V_{\mathrm{gs}}$ and $V_{\mathrm{gi}}$ is inverse to that of a traditional noncollinear OPA. Equation (3) means that the signal pulse front has to be tilted to the idler propagation direction. The idler, when pumped by a picosecond pulse where its physical length is larger than its beam diameter, will simply acquire a pulse front that matches the signal pulse front [14]. If the signal pulse front is tilted to the idler propagation direction, the generated idler will possess no pulsefront tilting, that is, no angular dispersion [14]. It is slightly more complicated when a femtosecond pump pulse used since pump pulse-front tilting is also needed to keep the idler free from angular dispersion [15].

To demonstrate the idea, we built a single-stage OPCPA generating broadband, angular-dispersion-free $2.15 \mu \mathrm{m}$ idler pulses. In this example, $V_{\mathrm{gs}}>V_{\mathrm{gi}}$, and thus neither collinear geometry nor traditional noncollinear geometry can be used to achieve broad phase-matching bandwidth. Figure 2 shows the schematic diagram of the setup. It starts with an octave-spanning Ti:sapphire oscillator (Idesta QE). A $1 \mathrm{kHz}$ Nd:YLF chirped pulse amplifier (CPA) [16] was injection-seeded by the $1047 \mathrm{~nm}$ spectral component of the oscillator for the optical synchronization. A small fraction of the Nd:YLF CPA output was used to generate the $8 \mathrm{ps}, 120 \mu \mathrm{J}, 523 \mathrm{~nm}$ pump pulses by second-harmonic generation in an LBO crystal. The remaining oscillator output was spatially chirped by a grating pair before being focused by an achromatic lens (ACL). The grating separation determines the angular dispersion applied to the signal. A Brewster prism pair can also be used to introduce the required angular dispersion, but the performance is inferior because it gives too much higher order dispersion compared to typical material dispersion. The available signal pulse energy for

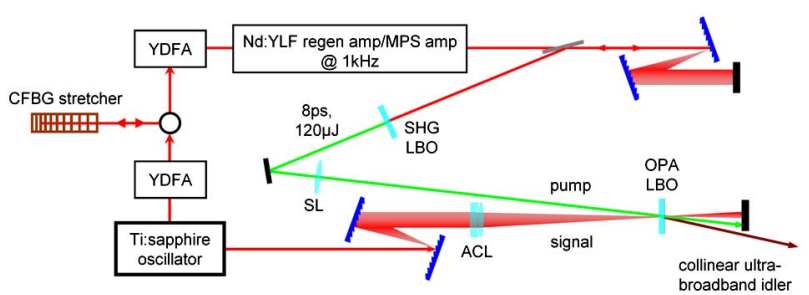

Fig. 2. (Color online) Schematic diagram of the experimental setup. YDFA, ytterbium-doped fiber amplifier; CFBG, chirped fiber Bragg grating; MPS amp, multipass slab amplifier. seeding the OPCPA was $0.25 \mathrm{~nJ}$. A single-stage OPCPA with a gain of 2000 is constructed using a $10 \mathrm{~mm}$ type-I LBO crystal.

Figure 3 presents the far-field idler spectra. Three spectra taken at different transverse positions of the beam are shown for each experimental condition. The baseline result, obtained with $\alpha=1^{\circ}$ and $d \alpha d \lambda=0$, is shown in Fig. 3(a). As expected, the amplification bandwidth is narrow because there is no solution to the condition for the traditional noncollinear OPA. Figure 3(b) shows the result when the signal angular dispersion was properly applied, but the noncollinear angle was not set correctly $\left(\alpha=16^{\circ}\right.$ and $\left.d \alpha d \lambda=100 \mu \mathrm{rad} / \mathrm{nm}\right)$. Figure 3(c), on the other hand, shows the result when the noncollinear angle was set correctly, but the signal angular dispersion was not properly applied $\left(\alpha=1.0^{\circ}\right.$ and $d \alpha / d \lambda=64 \mu \mathrm{rad} / \mathrm{nm}$ ). In either condition, the amplification bandwidth is increased compared to Fig. 3(a). However, the spatial chirp indicates that idler angular dispersion is present. This is not surprising, since the condition of broadband amplification [Eq. (2)] can still hold if one would allow idler angular dispersion $(d \beta / d \omega \neq 0)$. In fact, it has been previously shown that an angularly dispersed signal can be employed to extend the phase-matching bandwidth of a near-IR OPCPA [17-19]. However, it is only when both conditions in Eq. (3) are fulfilled $\left(\alpha=1.0^{\circ}\right.$ and $\left.d \alpha d \lambda=100 \mu \mathrm{rad} / \mathrm{nm}\right)$ that one can obtain a broadband, angular-dispersion-free idler as illustrated in Fig. 3(d). The centroid of the spectra remains the same across the beam, showing no measurable angular dispersion. The inset shows the far-field beam profile, showing low beam ellipticity of less than 1.1.

The generated broadband, angular-dispersion-free idler can be used, for example, for the seeding of a high-energy $2.15 \mu \mathrm{m}$ OPCPA for long-wavelength driven HHG [6]. Generally, the weak seed, a few picojoules, limits the amplified output energy of the current $2.15 \mu \mathrm{m}$
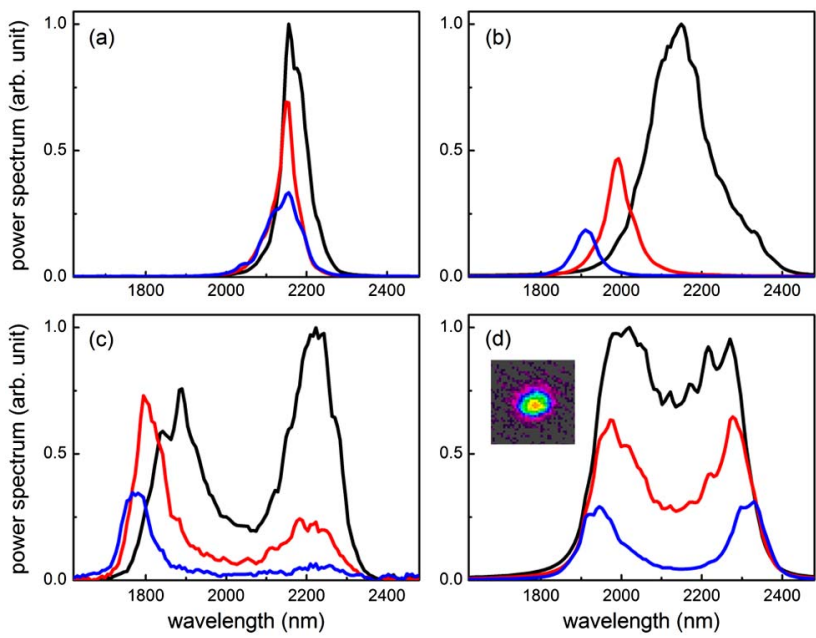

Fig. 3. (Color online) Far-field idler spectra measured at different transverse positions of the beam (black, red, and blue) under different experimental conditions. (a) $\alpha=1.0^{\circ}$ and $d \alpha / d \lambda=0 ; \quad$ (b) $\quad \alpha=1.6^{\circ} \quad$ and $\quad d \alpha d d \lambda=100 \mu \mathrm{rad} / \mathrm{nm}$; (c) $\alpha=1.0^{\circ}$ and $d \alpha / d \lambda=64 \mu \mathrm{rad} / \mathrm{nm} ;$ (d) $\alpha=1.0^{\circ}$ and $d \alpha^{\prime} d \lambda=100 \mu \mathrm{rad} / \mathrm{nm}$. Inset: the far-field beam profile. 

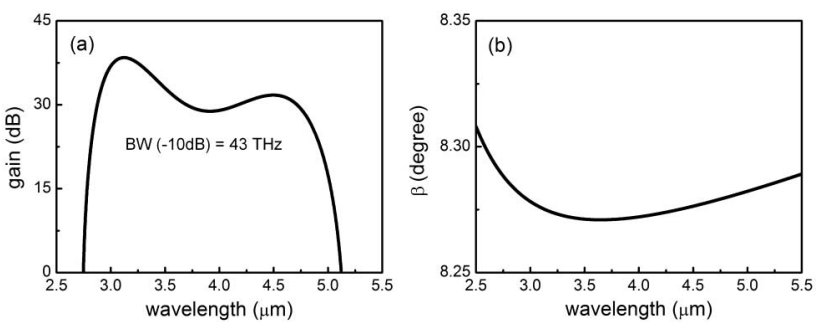

Fig. 4. Simulation results of an OPCPA utilizing the proposed concept. A bandwidth close to an octave, centered at $3.5 \mu \mathrm{m}$, can be obtained (a) with negligible angular dispersion (b). Such a bandwidth supports a 1.6 cycle (19 fs) pulse.

OPCPA because of the pump depletion by superfluorescence $[16,20]$. It has been a recent endeavor to improve the $2 \mu \mathrm{m}$ seed power $[13,21]$. Starting with the scheme shown here, the effective seed has a photon number equal to the phase-matched signal band of the Ti:sapphire oscillator (nanojoule-level energy), and thus the initial signal-to-noise ratio is improved by two orders of magnitude [22].

Figure 4 shows another example of our scheme, applied to the 3-5 $\mu \mathrm{m}$ regime. Applying the local approximation, we have used the analytic formulas for describing the gain of an OPCPA [23,24]. In a $1 \mathrm{~mm}$ periodically poled lithium niobate crystal with a pump wavelength of $1.03 \mu \mathrm{m}$ and a poling period of $22.8 \mu \mathrm{m}$, Eq. (3) can be satisfied by setting $\alpha=4.1^{\circ}$ and $d \alpha d \lambda=145 \mu \mathrm{rad} / \mathrm{nm}$. The whole system is compatible with $\mathrm{Yb}$ fiber technology or $\mathrm{Yb}$ :YAG laser technology as the signal (1.25-1.85 $\mu \mathrm{m})$ can also be generated by super-continuum generation in a photonic crystal fiber [25]. The calculation shows that a bandwidth of $43 \mathrm{THz}(-10 \mathrm{~dB})$ centered at $3.5 \mu \mathrm{m}$ can be obtained with negligible angular dispersion (200 $\mu \mathrm{rad}$ across the full amplification bandwidth). The transform-limited pulse duration of such a bandwidth is $19 \mathrm{fs}$ (1.6cycle), more than a factor of 2 shorter than the state-of-the-art, $1 \mu \mathrm{m}$ pumped, 3-5 $\mu \mathrm{m}$ OPCPA pulses $[\underline{9}, 10]$.

In conclusion, we present a new type of noncollinear OPA for direct generation of broadband, angulardispersion-free mid-IR idler pulses. A broadband (1.8-2.4 $\mu \mathrm{m})$, angular-dispersion-free idler pulse was generated experimentally and can be directly used to seed the $2.15 \mu \mathrm{m}$ OPCPA demonstrated in $[6,13]$. A calculation shows that the scheme can generate a broadband $3.5 \mu \mathrm{m}$ idler, with the bandwidth supporting a subtwo-cycle pulse. The scheme may also find applications in high-energy pulse synthesis [26]: a conventional nearIR OPCPA geometry could be used in preamplification stages, followed by a final power amplification stage utilizing our scheme to generate a broadband idler. Thus, a single system with two power amplifiers could be used to generate near-IR signal and mid-IR idler pulses, both broadband and angular dispersion free. The minimized uncommon path would simplify the required feedback loops and improve the system stability.
This work has been supported by Air Force Office of Scientific Research grant FA9550-10-1-0063 and the Center for Free-Electron Laser Science.

\section{References}

1. S. Woutersen, U. Emmerichs, and H. J. Bakker, Science 278, 658 (1997).

2. M. Bonn, C. Hess, S. Funk, J. H. Miners, B. N. J. Persson, M. Wolf, and G. Ertl, Phys. Rev. Lett. 84, 4653 (2000).

3. C. I. Blaga, F. Catoire, P. Colosimo, G. G. Paulus, H. G. Muller, P. Agostini, and L. F. DiMauro, Nature Phys. 5, 335 (2009).

4. M.-C. Chen, P. Arpin, T. Popmintchev, M. Gerrity, B. Zhang, M. Seaberg, D. Popmintchev, M. M. Murnane, and H. C. Kapteyn, Phys. Rev. Lett. 105, 173901 (2010).

5. A. D. Shiner, B. E. Schmidt, C. Trallero-Herrero, H. J. Wörner, S. Patchkovskii, P. B. Corkum, J.-C. Kieffer, F. Légaré, and D. M. Villeneuve, Nature Phys. 7, 464 (2011).

6. K.-H. Hong, S.-W. Huang, J. Moses, X. Fu, C.-J. Lai, G. Cirmi, A. Sell, E. Granados, P. Keathley, and F. X. Kärtner, Opt. Express 19, 15538 (2011).

7. D. Brida, C. Manzoni, G. Cirmi, M. Marangoni, S. De Silvestri, and G. Cerullo, Opt. Express 15, 15035 (2007).

8. D. Brida, M. Marangoni, C. Manzoni, S. De Silvestri, and G. Cerullo, Opt. Lett. 33, 2901 (2008).

9. C. Heese, C. R. Phillips, L. Gallmann, M. M. Fejer, and U. Keller, Opt. Lett. 35, 2340 (2010).

10. O. Chalus, A. Thai, P. K. Bates, and J. Biegert, Opt. Lett. 35, 3204 (2010).

11. G. Cerullo and S. De SilvestriRev. Sci. Instrum.74, 1 (2003).

12. K. Okamura and T. Kobayashi, Opt. Lett. 36, 226 (2011).

13. X. Gu, G. Marcus, Y. Deng, T. Metzger, C. Teisset, N. Ishii, T. Fuji, A. Baltuška, R. Butkus, V. Pervak, H. Ishizuki, T. Taira, T. Kobayashi, R. Kienberger, and F. Krausz, Opt. Express 17, 62 (2009).

14. O. Isaienko and E. Borguet, J. Opt. Soc. Am. B 26, 965 (2009).

15. A. Zaukevičius, V. Jukna, R. Antipenkov, V. Martinėnaitė, A. Varanavičius, A. P. Piskarskas, and G. Valiulis, J. Opt. Soc. Am. B 28, 2902 (2011).

16. J. Moses, S.-W. Huang, K.-H. Hong, O. D. Mücke, E. L. Falcão-Filho, A. Benedick, F. Ö. Ilday, A. Dergachev, J. A. Bolger, B. J. Eggleton, and F. X. Kärtner, Opt. Lett. 34, 1639 (2009).

17. G. Arisholm, J. Biegert, P. Schlup, C. Hauri, and U. Keller, Opt. Express 12, 518 (2004).

18. O. Isaienko and E. Borguet, Opt. Express 16, 3949 (2008).

19. K. Yamane, T. Tanigawa, T. Sekikawa, and M. Yamashita, Opt. Express 16, 18345 (2008).

20. T. Fuji, N. Ishii, C. Y. Teisset, X. Gu, T. Metzger, A. Baltuška, N. Forget, D. Kaplan, A. Galvanauskas, and F. Krausz, Opt. Lett. 31, 1103 (2006).

21. J. Moses, H. Suchowski, and F. X. Kärtner, Opt. Lett. 37, 1589 (2012).

22. C. Manzoni, J. Moses, F. X. Kärtner, and G. Cerullo, Opt. Express 19, 8357 (2011).

23. I. N. Ross, P. Matousek, G. H. C. New, and K. Osvay, J. Opt. Soc. Am. B 19, 2945 (2002).

24. J. Moses and S.-W. Huang, J. Opt. Soc. Am. B 28, 812 (2011).

25. T. Schreiber, J. Limpert, H. Zellmer, A. Tünnermann, and K. P. Hansen, Opt. Commun. 228, 71 (2003).

26. S.-W. Huang, G. Cirmi, J. Moses, K.-H. Hong, S. Bhardwaj, J. R. Birge, L.-J. Chen, E. Li, B. J. Eggleton, G. Cerullo, and F. X. Kärtner, Nat. Photon. 5, 475 (2011). 